\title{
Aerodynamic Design and Development of Infiniti 4.0 Solar Car using CFD
}

\author{
Tanmay Chitnis*,Navoday Borkar*, Sandesh Kumawat*,Vivek Adepu*, \\ *Student, \\ Department of Mechanical Engineering, \\ Sinhgad College of Engineering(SCoE), \\ Pune.
}

\begin{abstract}
Aerodynamic characteristics of a solar vehicle is of significant interest and importance to reduce the air drag experienced in order to reduce the power loss in overcoming various resistances. This paper deals with virtual wind tunnel testing using CFD and finding out the best possible design with due respect to the ESVC'2020 rulebook. A three dimensional CAD model of the vehicle was created using SolidWorks 2018.The model was meshed and CFD was performed subsequently in the ANSYS FLUENT. The drag coefficient was found to be about 0.697 at a speed of $72 \mathrm{Kmph}(20 \mathrm{~m} / \mathrm{s})$ due to large wake region formed behind the main roll hoop. Further reduction in drag coefficient is exhaustive as the main roll hoop is mandatory according to the rulebook.
\end{abstract}

Keywords-ANSYS, drag coefficient, CFD, main roll hoop, solid works, wake region, etc.

\section{INTRODUCTION}

Infiniti 4.0 is a four-wheel vehicle designed and fabricated by Team Hyperion, the official team of the department of mechanical engineering, SCoE,Pune.The team was formed in 2016 with a vision of becoming the trendsetters of the solar vehicle designers and manufacturers. It participates in various national level competitions like Electric Solar Vehicle Championship(ESVC), National Solar Vehicle Challenge (NSVC), etc. The Infiniti 4.0 has been designed in accordance with the guidelines dictated by the ESVC 2020 rulebook. [1]

In the recent past years, the world has realized the problem of energy crisis due to depleting fossil fuels, increasing population, etc. leading to deadly problems of ever increasing pollution and global warming. Automobiles are the most used means of transportation despite being the primary contributors to the global air pollution. Hence it was very logical for the scientists and researchers to find an alternative fuel to power the modern vehicles. [2]

It is a well understood fact that the future of mobility is solar electric hybrid vehicles, that being said the cost of energy produced by solar arrays remains on a higher side as compared to energy produced by nuclear fuels, coal, petroleum etc. [3] This high cost coupled with comparatively low efficiency of solar arrays makes it necessary to utilize the energy judiciously.

Given that almost $70 \%$ of all the power from the solar array is used to overcome the air resistance, aerodynamics of the solar power vehicle has to be given prime importance while designing the vehicle to minimize the power loses. Computational Fluid Dynamics (CFD) is a tool to study the fluid flow at minimum cost and provides sufficient accuracy with an average error less than $10 \%$. Hence CFD was used to optimize the design. [4]

\section{CFD THEORY AND IMPORTANCE}

Computational Fluid Dynamics (CFD) is the implementation of numerical methods using computational techniques to study the fluid flow and solving the mathematical equations which describe the behavior of fluids. [5] CFD is becoming increasingly popular in the fields of Heat Transfer and Applied Mechanics due to its fair capacity of predicting the motion of fluids and chemical reactions as well. Navier-Stokes equations are the governing equations to model the behavior of viscous fluids. As mentioned below, Navier-Stokes are generally represented in the partial differential equation form. The solving of these equations analytically using the usual various standard methods available to solve the partial differential equations for simple flows over simple geometries like laminar flow over a thin plate is possible but if the flow is to become turbulent over much of a complex body/geometry it is almost impossible to even get close to the actual solution .Hence, a time averaged Navier-Stokes is being used in conjunction with the various turbulence models to solve the issues resulting from the time averaging process .The Navier stokes equation which is the combination of two equations.[6] i.e.

\section{A) Continuity equation in $3 D$}

The unsteady three dimensional mass conservation or continuity equation at a point in compressible fluid can be represented as

$$
\frac{\partial \rho}{\partial t}+\operatorname{div}(\rho . \bar{u})=0
$$

The first term on the left side is Rate of change of density with respect to time. The second term describes the net flow of Mass across boundaries.

\section{B) Momentum equations in $3 D$}

The unsteady, momentum equation in $3 \mathrm{D}$ at a point in compressible fluid flow can be represented as

$$
\begin{aligned}
& \frac{\partial(\rho u)}{\partial t}+\operatorname{div}(\rho u \bar{u})=-\frac{\partial \rho}{\partial x}+\operatorname{div}(\mu \operatorname{grad}(u))+S_{M X} \\
& \frac{\partial(\rho v)}{\partial t}+\operatorname{div}(\rho v \bar{u})=-\frac{\partial \rho}{\partial y}+\operatorname{div}(\mu \operatorname{grad}(v))+S_{M Y} \\
& \frac{\partial(\rho w)}{\partial t}+\operatorname{div}(\rho w \bar{u})=-\frac{\partial \rho}{\partial z}+\operatorname{div}(\mu \operatorname{grad}(w))+S_{M Z}
\end{aligned}
$$


In each of these equation the first term on left side is the Rate of change of mass velocity with respect to time and the second term represents the convective momentum transfer. The first term on right side represents the pressure gradient in that particular direction. The second term represents the diffusive momentum transfer and the third term represents the source.

Various models are considered to solve the Navier stokes equation. The turbulence model analyzed in the CFD simulation should be able to capture the main flow effects around the vehicle. Different turbulence model is studied in simulation ranging from steady state approaches, $k-\varepsilon$ and $k-\Omega$ SST models, to transient models like DDES i.e. Delayed Detached eddy simulation. For the RANS portion, the turbulence model considered are $\mathrm{k}-\varepsilon$ and $\mathrm{k}-\Omega$ models. [6]

The $k-\varepsilon$ turbulence model is composed by two equations that come directly from the differential transport equation, in which $\mathrm{k}$ represents turbulence kinetic energy and $\varepsilon$ represents the turbulence dissipation rate. The two equations are mentioned below

$$
\frac{\partial \rho k}{\partial t}+\operatorname{div}\left(\rho k u_{r}\right)=\operatorname{div}\left(\frac{\mu_{t}}{\sigma_{k}} \operatorname{grad} k\right)+2 c E_{i j} E_{i j}-
$$

$$
\begin{array}{r}
\frac{\partial \rho \varepsilon}{\partial t}+\operatorname{div}\left(\rho \varepsilon u_{r}\right)=\operatorname{div}\left(\frac{\mu_{t}}{\sigma_{\varepsilon}} \operatorname{grad} \varepsilon\right)+C_{1 z} \frac{\varepsilon}{k} 2 \mu_{t} E_{i j} E_{i j}- \\
C_{2 z} \rho \frac{\varepsilon^{2}}{k}
\end{array}
$$

Where $\mu_{t}$ is the turbulent viscosity, $C_{1 z}, C_{2 z}, \sigma_{\varepsilon}$ and $\sigma_{k}$ are the turbulence model constants.

The k- $\Omega$ turbulence model is composed by two equations, in which $\mathrm{k}$ represents the turbulence kinetic energy and $\Omega$ represents the specific dissipation rate. The k- $\Omega$ SST model combines the advantages of $\mathrm{k}-\varepsilon$ and $\mathrm{k}-\Omega$ models. The two equations are mentioned below

$\frac{\partial \rho k}{\partial t}+\operatorname{div}\left(\rho k u_{r}\right)=\operatorname{div}\left(\frac{\mu_{t}}{\sigma_{k}} \operatorname{grad} k\right)+G_{k}-Y_{k}+S_{k}$

$$
\frac{\partial \rho \Omega}{\partial t}+\operatorname{div}\left(\rho \Omega u_{r}\right)=\operatorname{div}\left(\frac{\mu_{t}}{\sigma_{\Omega}} \operatorname{grad} \varepsilon\right)+G_{\Omega}-Y_{\Omega}+D_{\Omega}+
$$

Where $\mu_{t}$ is the turbulent viscosity,$\sigma_{\Omega}$ and $\sigma_{\Omega}$ are the turbulence model constants, $G_{k}$ represents the turbulent kinetic energy, $G_{\Omega}$ represents the generation of $\Omega, Y_{k}$ and $Y_{\Omega}$ represent the dissipation of and $\Omega$ due to turbulence, $D_{\Omega}$ represents the cross diffusion term, $S_{k}$ and $S_{\Omega}$ are user defined source terms.

\section{C) Non Equilibrium wall function (NWF):}

For high Reynolds number flow, such as external flow around vehicle resolving the near wall region down to the wall is not practical. NWF takes into account the effect of local variation of viscous sub layer, when computing the turbulent kinetic energy budget in wall adjacent cells. Also, NWF focuses on adverse pressure gradient region which are common in fluid flow around vehicles. NWF provides more realistic prediction of the turbulent boundary layer, including flow separation, and they do it without increasing the computational time. [9]

So, in the present solution, $\mathrm{k}-\Omega$ turbulence model with nonEquilibrium wall function is implemented to study the fluid flow around the vehicle.

\section{D) Inlet condition calculation}

Scale $=1: 5$

Actual length $=2.5 \mathrm{~m}$

Assumed velocity $=20 \mathrm{~m} / \mathrm{s}$

$\operatorname{Density}(\rho)$ of air $=1.225 \mathrm{~kg} / \mathrm{m}^{3}$

Viscosity $(\mu)$ of air $=1.8 \mathrm{e}-5$ Pa. $s$

Actual Reynolds no. Calculation

$$
\begin{aligned}
R_{E} & =\frac{\rho * v * l}{\mu} \\
& =\frac{1.225 * 20 * 2.5}{1.8 * 10^{-5}} \\
& =3402777.77
\end{aligned}
$$

Velocity calculation for inlet condition for scaled down geometry,

$R_{E}=3402777.77$

Scale down length $=0.5 \mathrm{~m}$

$$
\begin{aligned}
R_{E} & =\frac{\rho * v * l}{\mu} \\
v & =\frac{3402777.77 * 1.8 * 10^{-5}}{1.225 * 0.5} \\
v & =100 \mathrm{~m} / \mathrm{s}
\end{aligned}
$$

\section{METHODOLOGY}

\section{A. CAD Modelling}

CAD (Computer Aided Design) model of the solar vehicle is prepared in CAD software SolidWorks as shown in Fig. 1. The prepared design was as per the constraints given in the rulebook like limits of track width, wheel base, maximum area for solar panel, height of roll hoop, etc.

Also, the parameters like aesthetics, driver ergonomics are taken into consideration while designing. The limits for the maximum length, width and height are 100 inch, 60inch and 60 inches respectively as per the rulebook. [1] 


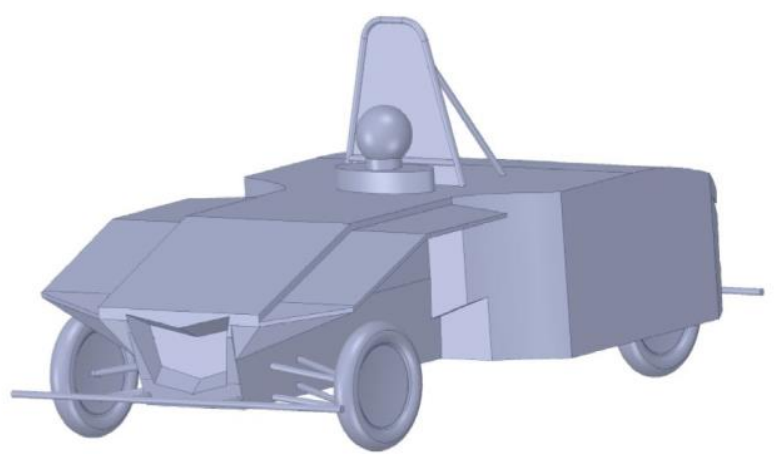

Fig. 1. 3-D CAD Model of Solar Vehicle

\section{B. Geometry Preparation}

Once the CAD Model is ready, it is very important to create a numerically acceptable geometry for the solver to perform CFD Analysis.

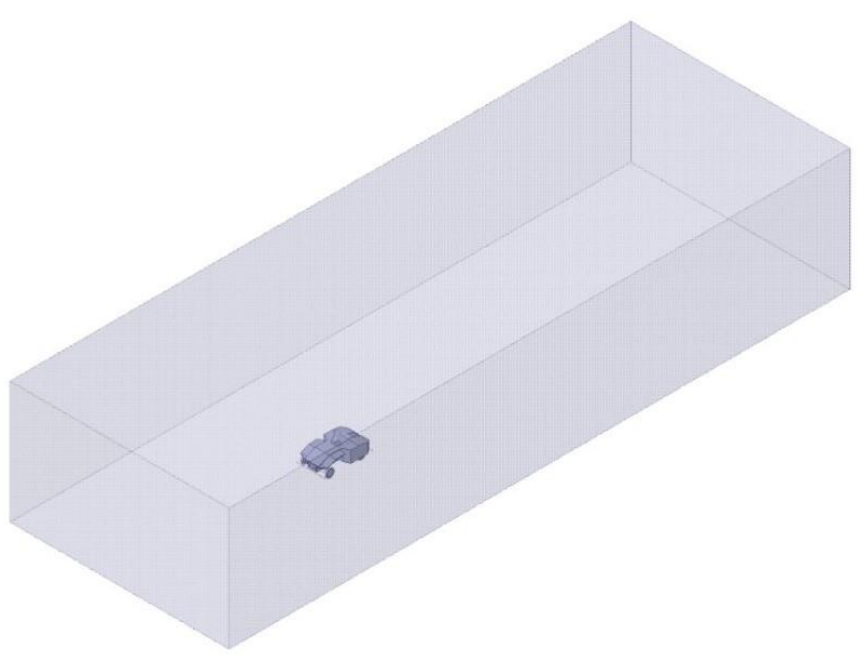

Fig. 2. Enclosure

It was observed that the computational time required for the simulation of the original geometry was unreasonable. Hence, performing CFD simulation of the original model for number of iterations was not a feasible solution and model for CFD simulation was scaled down in 1:5 ratio.

Once the model is scaled down, enclosure as a computational domain as shown in Fig. 2. was prepared for the simulation. Width and height of the domain is 1 meter from the car which can be observed in Fig. 3. The length of the domain is 4 meters behind the car and 2 meters in front of the car as shown in Fig. 4. The purpose of providing maximum length behind the car is to ensure that the wake behind the car is properly solved before exit plane

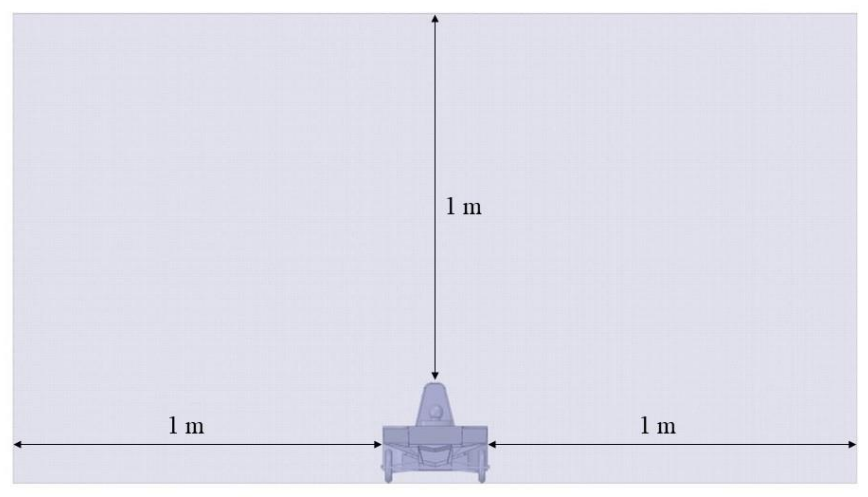

Fig. 3. Front View of Enclosure

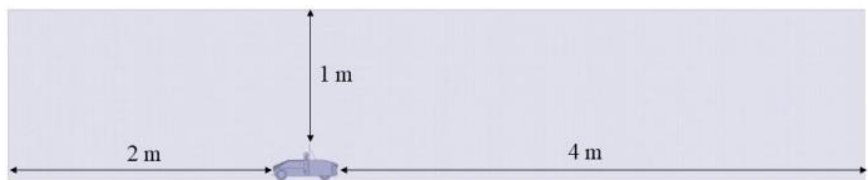

Figure 4 Side view of Enclosure

\section{Meshing}

After the Geometry was reconstructed and became numerically acceptable, the file was imported into ANSYS, to construct the three-dimensional Grid system. Tetrahedral Unstructured Mesh cells were used in the computational Domain.

For the above grid system, the cell size gradually grows from around $3.3406 \mathrm{e}-003 \mathrm{~m}$ near the car surface up to about $0.2 \mathrm{~m}$ in the far-field where the incoming flow is assumed to be undistributed by the presence of the car. The Grid density is high around the car where the flow gradients can be large (Fig. 5.). For the same reason, there are also densely pack mesh near the car surface which can be observed from Fig. 6. There are 234182 nodes and 814627 elements in the grid.

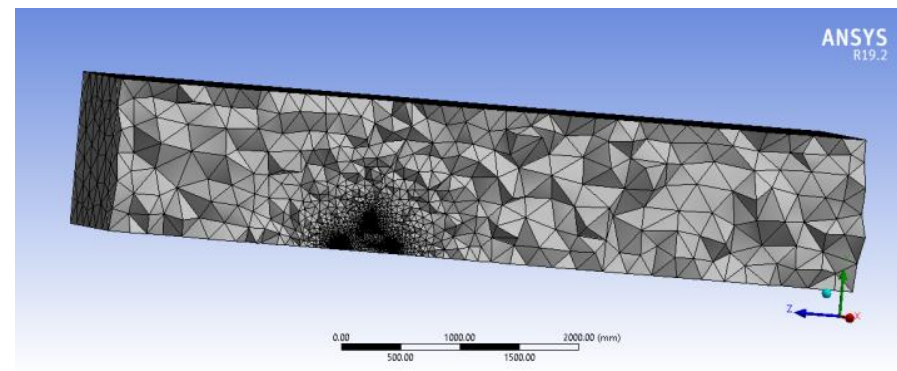

Fig. 5. Meshing of Enclosure

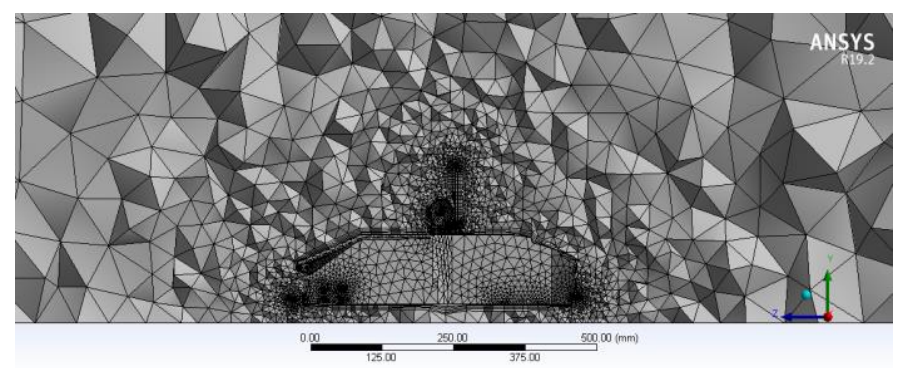

Fig. 6. Meshing 
For the geometry, inflation feature has been setup to utilize the growth of 5 layers from the surface of car boundary with total thickness of $12.5 \mathrm{~mm}$ and 1.2 growth rate from the first layer. In addition to capturing the boundary layer effect accurately, inflation also contributes to lesser element count and computational time. Inflation layer are clearly visible in Fig. 7. around the surface of solar vehicle.

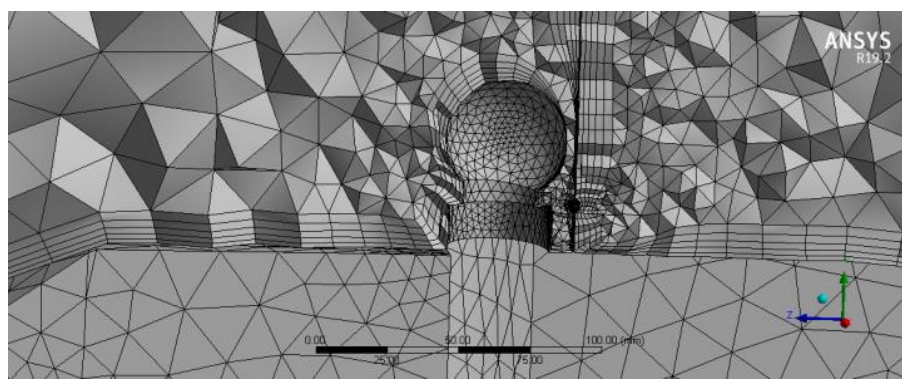

Figure 7 Inflation layers

\section{Boundary Conditions}

The front plane was given the "velocity-inlet" boundary condition. The bottom of the domain and solar vehicle was set as "No slip wall" and the top and the sides of the domain are set as "Symmetry" so that these surfaces would not bring any effects on the simulation. The outlet boundary condition is set to "Pressure-outlet" with gauge pressure of 0 Pascal. All the boundaries can be observed in Fig. 8 .

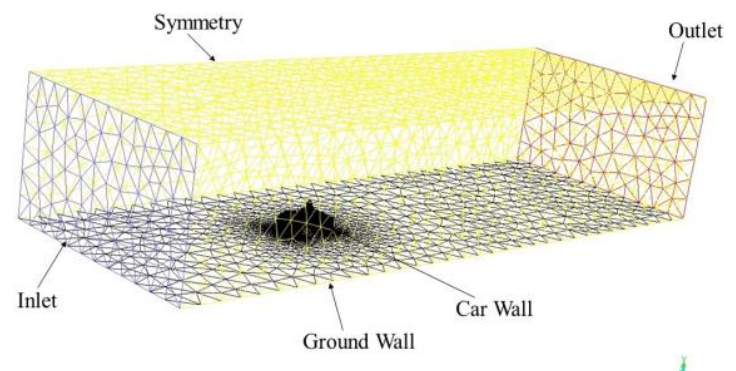

Fig. 8. Boundary Conditions for Enclosure

In Fluent, the three dimensional, segregated, steady state solver is used. Air was selected as working fluid with density of $1.225 \mathrm{~kg} / \mathrm{m}^{3}$. From the calculations, reference values for area, length, velocity and viscosity were given as 0.05422371 $\mathrm{m}^{2}, 0.5 \mathrm{~m}, 100 \mathrm{~m} / \mathrm{s}$ and $1.8 \mathrm{e}-05$ respectively for the value of Reynolds number to be constant. In addition to the Residuals of numerically solved governing equations, monitoring of the lift coefficient $C_{l}$ and the drag coefficient $C_{d}$ to determine the convergence of the computations has been done.

\section{E. Simulation Results}

The numerical simulation was solved for 300 iterations. The changes in the graphs for each iteration were observed and was considered converged as there was recurrence of same fluctuation after 100 iterations till 300 iterations. The graph of Residuals can be observed in Fig. 9.

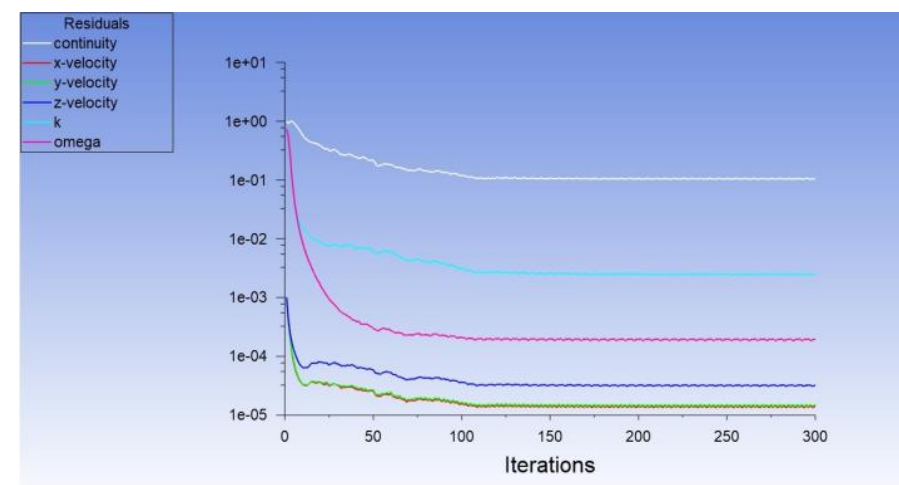

Fig. 9. Graph of Residuals

The numerical drag coefficient evolutions during the iterations can be observed in Fig.10. The final value of drag coefficient computed is 0.69743 .

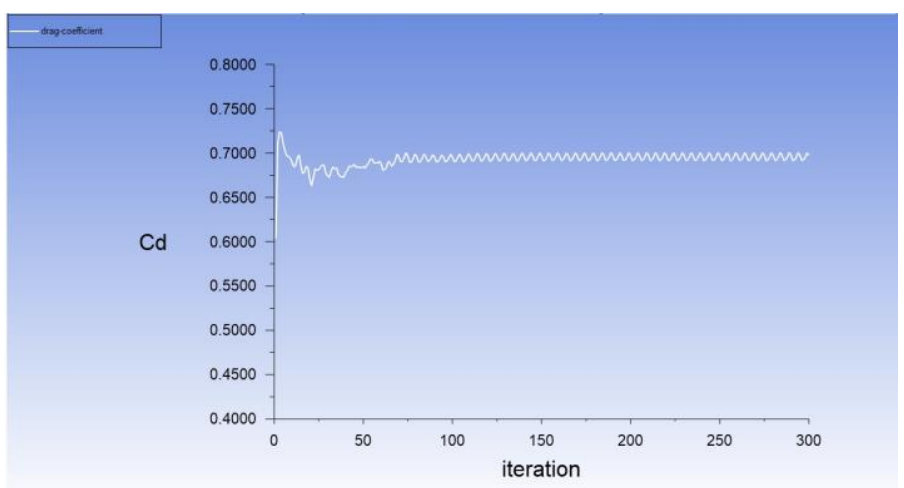

Fig. 10. Drag Coefficient Plot

The graphical change in lift coefficient during the iterations can be observed in Fig.11. The positive value of 'coefficient of lift' means an upward force is exerted on the vehicle. The value of lift was selected considering the available traction, rolling resistance offered due to combined effect of weight and lift force. The value of lift coefficient after simulation is 0.08273 .

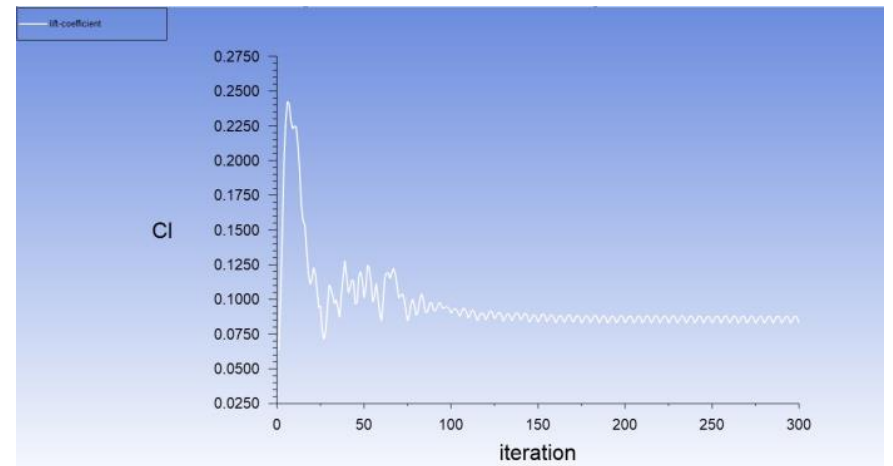

Fig. 11. Lift Coefficient Plot 
The pressure distributions on the surfaces of the car has been examined and presented in Fig. 12. The regions of high pressure appear at the frontal area and the roll hoop of the vehicle. The airflow experiences resistance at these locations, hence it is expected that the pressure increases when velocity drops. Also, we can see that the flow field around the whole car surfaces develops in a relatively smooth manner expect for the frontal area and roll hoop.

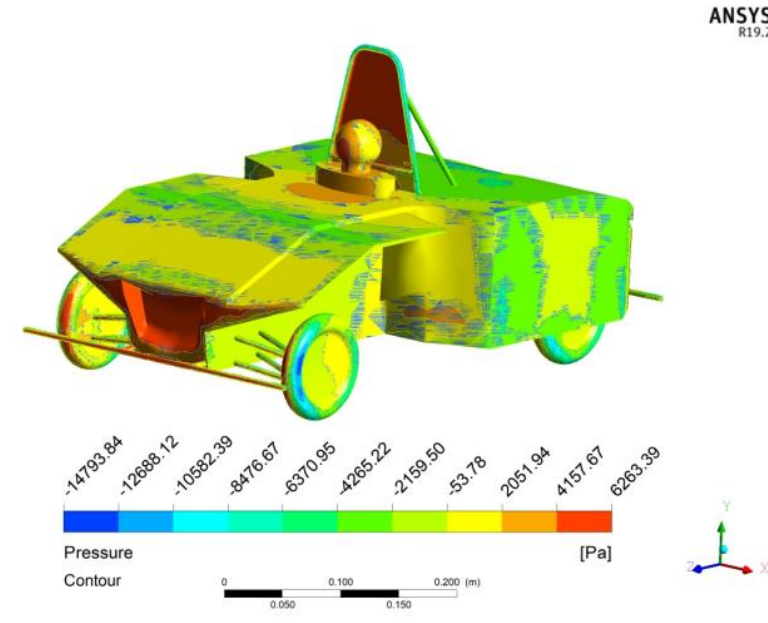

Fig. 12. Pressure Contours

Velocity vectors and streamlines can be observed in Fig. 13. and Fig. 14. respectively at mid-plane of vehicle. They provide an excellent visualization of the flow around the solar vehicle depicting details of wake region. Wake region is the most important thing to analyze in order to determine amount of drag and lift experienced by the body.

Wake region can be observed behind the roll hoop which shows the separation of boundary layer due to adverse pressure gradient. Due to the increase in pressure, velocity behind the roll hoop is minimum which can be observed clearly from velocity contours at mid-plane of solar vehicle in Fig. 15.

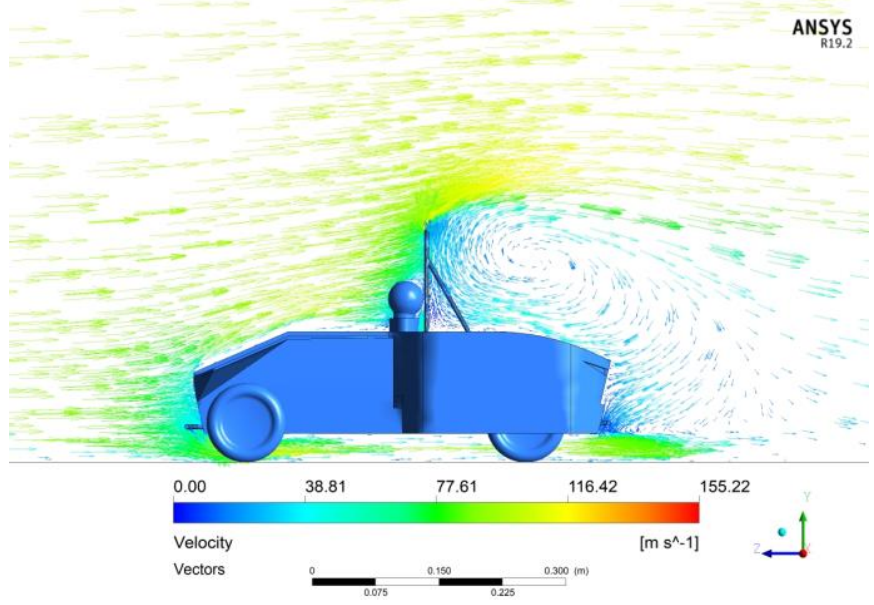

Fig. 13. Velocity Vectors

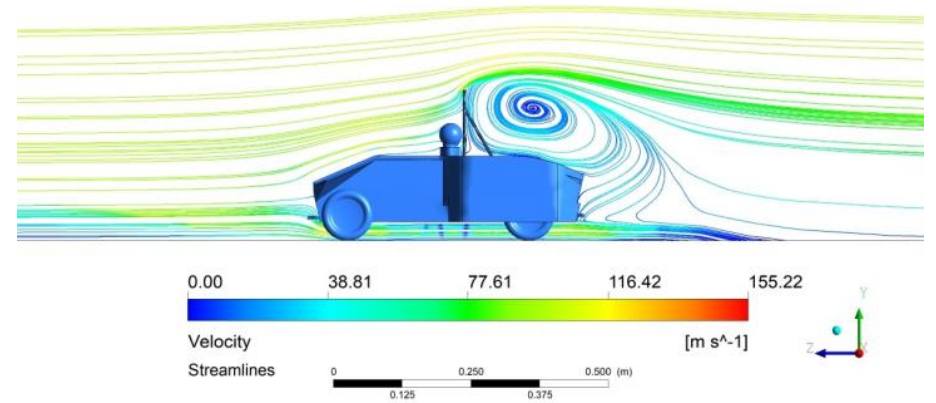

Fig. 14. Velocity Streamlines

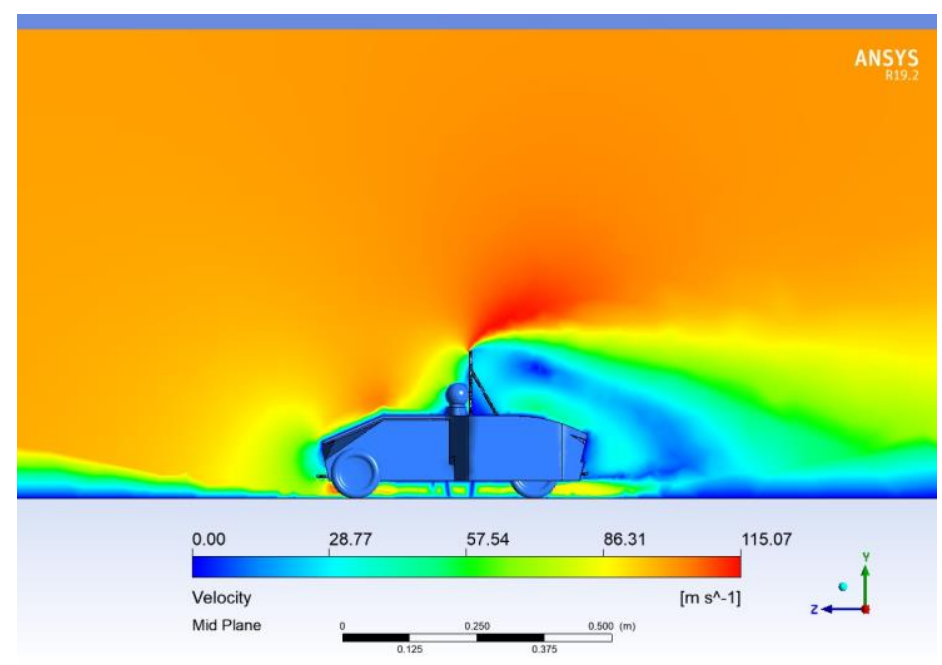

Fig. 15. Velocity Contours at Mid-plane

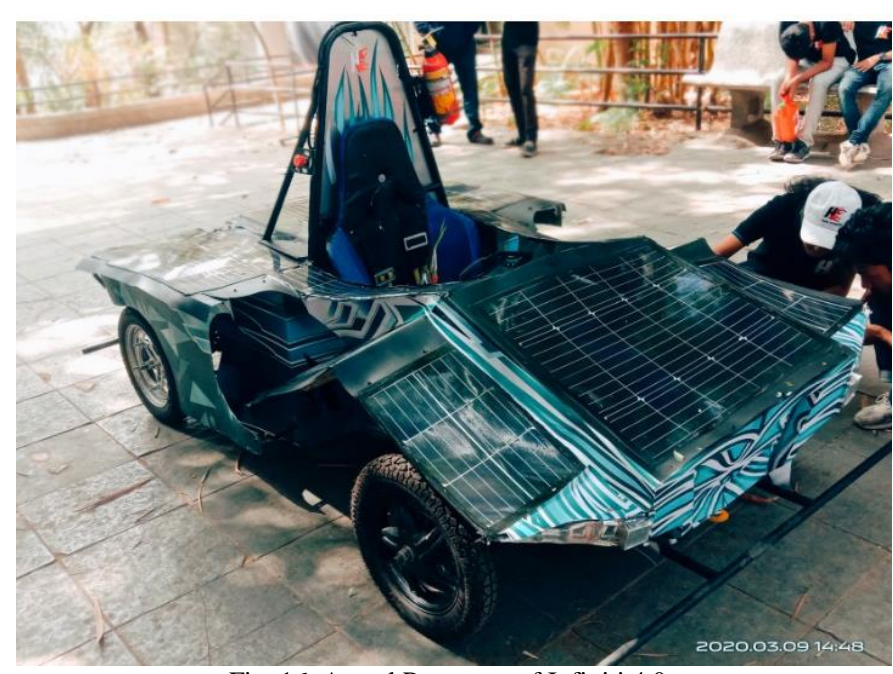

Fig. 16 .Actual Prototype of Infiniti 4.0

\section{CONCLUSION}

Fluid Flow around the vehicle was successfully studied around the car body using CFD. Designing of the vehicle aerodynamically is difficult as the guidelines of the competitions rulebook has to be strictly followed. As demonstrated in the above various graphics, wake region formed is comparatively very large as compared to other commercial vehicles and the main reason behind it was the roll hoop providing a large surface area to generate increased pressure drag. The drag coefficient was found to be 0.697 
which is more than many super cars. However, it was considered the best design with due respect to the rulebook's guidelines. Also, the actual working prototype was manufactured which is as shown in fig. 16.

\section{ACKNOWLEDGMENT}

The authors would like to thank Prof N. S. Gohel for her constant support and guidance. The authors would also like to acknowledge a sense of gratitude to Prof. V. N. kapatkar,Head of Department of Mechanical Engineering and Dr. S. D. Lokhande,principal ,SCoE,Pune for constant encouragement.

\section{REFERENCES}

[1] https://www.worldsolarproject.com/rulebook.

[2] Hucho W.H.,"Aerodynamics of Road Vehicles", $4^{\text {th }}$ edition, SAE Publishing1998.

[3] F.G. Rieck, C. Machielse , J.H.R van Duin ,"Automotive, the Future of Mobility", EVS30 Symposium,Stuttgart,Germany,October 911,2017 .
[4] Sam Paterson, Pujith Vijayaratham, Charith Parera and Graham Diog ," Design and development of the Sunswift eVe solar vehicle: a record-breaking electric car",2013.

[5] Senan Thabet, Thabit H. Thabit, "CFD Simulation of the Air Flow around a Car Model (Ahmed Body)", International Journal of Scientific and Research Publications, Volume 8, Issue 7, July 2018.

[6] Anderson CFD book: the basic with application McGraw-Hill series in aeronautical and aerospace engineering.

[7] Filipe Buscariolo,Flavio Maruyama ,Julio cesar Alves ,"Analysis of turbulence models Applied to CFD drag Simulation of Small Hatchback Vehicle", SAE Technical paper.

[8] David C. Wilcox ,'Turbulence Modelling for CFD book ", DCW Industries,Inc.

[9] Akshay Parab, Amar Sakarwala, Vaibhav Patil, "CFD analysis of the flow variation due to addition of aerodynamic devices on underbody of Renault Duster using Fluent”, IJERT 2015.

\section{ABBREVIATIONS AND ACRONYMS}

1) $\mathrm{CFD}=$ Computational Fluid Dynamics

2) $\mathrm{ESVC}=$ Electric Solar Vehicle Championship

3) $\mathrm{NWF}=$ Non Equilibrium wall function

4) RANS= Reynolds Average Navier Stokes Equation 